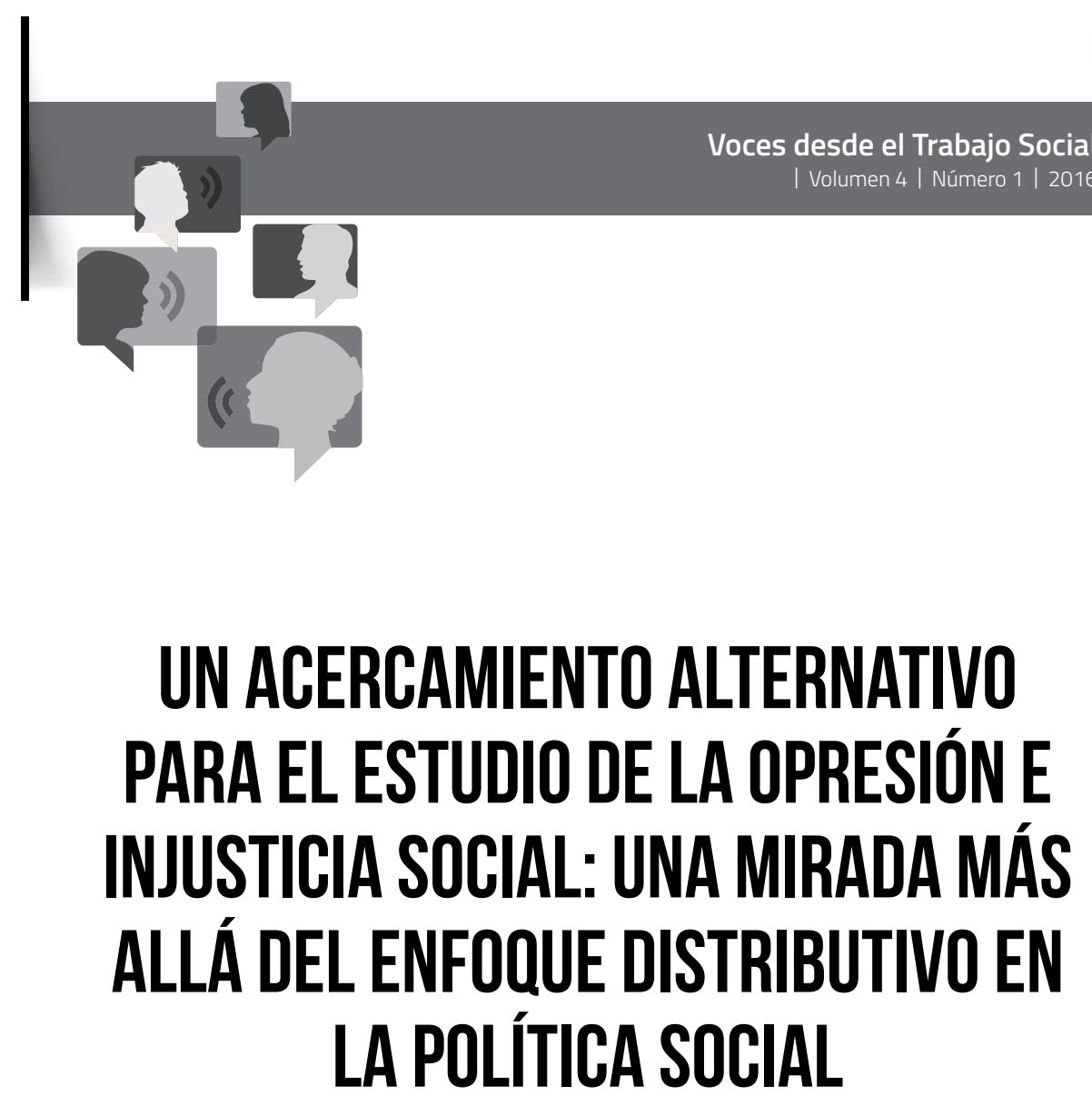




\section{Eduardo J. Zavala-Mendoza}

Posee bachillerato en Desarrollo

Socioeconómico y Ambiente (E.A.P.);

Certificado en Política y Ambiente del Global Enviroment Centre Foundation (Osaka);

Maestría en Planificación, siendo actualmente estudiante Doctoral en Trabajo Social (UPR/

RP). Ha sido director ejecutivo e investigador de ONG'scomunitarias.

\section{Recibido:}

1 de marzo de 2016

\section{Aprobado:}

31 de agosto de 2016 


\title{
UN ACERCAMIENTO ALTERNATIVO PARA EL ESTUDIO DE LA OPRESIÓN E INJUSTICIA SOCIAL: UNA MIRADA MÁS ALLÁ DEL ENFOQUE DISTRIBUTIVO EN LA POLÍTICA SOCIAL
}

\author{
Eduardo J. Zavala-Mendoza
}

\section{Resumen}

Se traza un tono afirmativo que expone en el centro del debate un [re]pensamiento de la opresión e injusticia social a partir de la conjunción: prejuicio más poder. Este acercamiento insumiso a cualquier enfoque lineal y reduccionista, permite vocalizar tramos de su recorrido, fuerza y pertinencia. Alejado del determinismo y subordinación a la docilidad, presento un entramado que marca la posibilidad de trascender las limitantes del paradigma distributivo, mismo que ha predominado en el análisis y configuración de la política social. Se sostiene que este enfoque es incapaz de asumir las propias implicaciones de su ejercicio: combatir la injusticia social y opresión. Para ello, se reconoce que dentro de este análisis resulta fundamental la consideración del ser humano como un sujeto inmerso en un entramado de relaciones de poder. A su vez, esto le 
sitúa dentro de múltiples ubicaciones e intensidades interseccionales de la opresión, las cuales tienen que ver con género, raza, edad, etnia, clase, nacionalidad, sexualidad, por mencionar algunos. En tal sentido, el presente ejercicio autoreflexivo no representa una locución verbal del dictamen de mi conciencia, sino que es el preludio de una ética que vista desde el Trabajo Social, permite trascender hacia la diversidad y que colectiviza el mundo con fuerza.

Descriptores: Opresión, justicia/injusticia social, política social, paradigma distributivo, prejuicio/ poder.

\section{Abstract}

The oppression and social injustice notion is exposed from an alternative view as outlined in the conjunction: prejudice plus power. In this effort, a theoretical framework is presented to construct the possibility of overcoming the limitations of the distributive approach, which has prevailed in the analysis and configuration of social policy aimed at addressing oppression and social injustice. It is argued that this approach is redistributive, reductionist and palliative, which is insufficient given the complexity of the study of oppression and social injustice. With this, the paradigm is unable to assume the implications of their own practice: combating social injustice and oppression. This examination begins with the study of the types of relations of power; but also recognizes 
the existence of multiple locations and intensities of intersectional oppression, which has to do with gender, race, age, ethnicity, class, nationality and sexuality. In that sense, this self-reflexive exercise does not represent a verbal utterance of the opinion of my conscience. This is a prelude to an ethic from Social Work which allows transcend towards diversity and unites (collectivized) the world with strength.

Keywords: Oppression, social justice/injustice, social policy, distributive paradigm, prejudice/power. 


\section{A manera de introducción}

Este es un volumen dedicado enteramente a presentar una óptica analítica que asume valores cónsonos con la justicia social, y que en tal tarea, genera un des-cubrir de diversas formas de opresión. En tal sentido, se recurre a una reflexión teórica y de la práctica de la política social capaz de apartarse de toda perspectiva que no considera la complejidad social y de su vasto espectro de relaciones intersubjetivas en la que se configuran las personas y los colectivos. Se espera que lo acá vertido pueda tener alguna utilidad en el análisis, elaboración e implantación de la política social.

Así, se provee de una contextualización crítica sobre algunos factores necesarios de ser problematizados y trascendidos a partir de lo entronizado y conformado desde el paradigma distributivo. Es decir, se intenta problematizar de que en el análisis de la injusticia social no solo las privaciones o inequidades en el plano de lo material deben considerarse, sino que también en la esfera de lo simbólico y en el ejercicio del poder. En tal sentido, se recurre a una facultad activa de la imaginación que posibilita la síntesis de lo múltiple [facultas imaginadi] para hacer presente lo que aparenta ausente.

Advierto que los temas que se abordan, son de tal dimensión compleja y matricial, lo que me orienta a nombrarles indicativamente, pero que no escapan y limitan a verterse desde una autenticidad creadora del Trabajo Social, como también desde la diversidad de la experiencia humana. Es decir, que desde el pensum y practicum propio del Trabajo Social se generan aprehensiones excepcionales y conectoras entre diversos lugares epistémicos, ideológicos y éticos. Por ende, en esta labor se constituye una o múltiples especificidades en las mediaciones de un modo particular de pensar, y que tiene como resultado, un hacer característico. 
Los aspectos anteriores, connotan de mayor relevancia cuando se yuxtaponen a un contexto de un sistema mundo que excluye, rechaza, niega y fragmenta. Con ello, no es de extrañar, requiere de acceder críticamente al pensamiento de diversos autores que connotan marcada relevancia para la argumentación que se presenta. En definitiva, esto conlleva a plantear dos frentes rizomáticos argumentativos, que se desarrollan a lo largo del escrito.

Primero, se pone en rumbo el pensar la opresión e injusticia social desde una perspectiva alternativa, que conducida por el duplo prejuicio y poder, justifique la necesidad de una visión más allá de lo distributivo. Esto es debido a que una política social vista únicamente desde este paradigma "será vista no como producto histórico, sino, como fruto de un desarrollo natural" (Pastorini, 1999, p. 213). En tal sentido, se afirma que este paradigma es conducente, pero no suficiente [o sea, es incapaz] para el abordaje de la opresión e injusticia social.

Segundo, ante tales interpretaciones urgidas anteriormente, se abre paso al análisis de cómo la interseccionalidad de diversos elementos y el poder configurado a partir de un sistema mundo colonial/moderno, articulan en su complementariedad una perspectiva que posibilita la comprensión de la opresión e injusticia social. Es decir, surgen argumentos que explican la existencia de múltiples ubicaciones e intensidades interseccionales de la opresión en el ser humano. También, se expone la influencia que impacta a todo sujeto a partir de una construcción hegemónica y de poder, elementos que son inscritos desde la modernidad y a partir de un aparente y único rostro ilustrado.

Cabe destacar que la mirada que acá se presenta, se vierte desde una visión transdisciplinaria y desde la experiencia de vida humana que construye, deconstruye y [re]edifica continuamente 
la fluidez de mi propia identidad. O sea, mi interpretación se enuncia desde una perspectiva dialógica configurada partir de ser hombre, hondureño, caribeño, latinoamericano, migrante, mestizo y trabajador social en formación.

Finalmente, es importante aclarar mi rechazo al uso sexista y androcéntrico del lenguaje. Por lo tanto, trataré en la mayor medida de recurrir a la narración combinada con términos colectivos y abstractos, formas masculinas y femeninas alternas, y que en el caso de usar la palabra en masculino, no implica una miopía que intenta borrar y naturalizarla ausencia de identidades de género.

\section{En relación al paradigma distributivo: conducente, pero no suficiente}

Preámbulo al paradigma distributivo.

La larga tradición teórica e ideológica que ha acompañado la definición de justicia social y opresión dentro de las políticas sociales, generalmente ha recaído sobre lo contenido en las políticas de la [re]distribución (Fraser, 1995). Estas políticas según Pastorini (1999), son formuladas desde una visión tradicional y son esbozadas como un conjunto de acciones por parte del Estado para disminuir las desigualdades sociales desde la perspectiva redistributiva. O sea, estas son vistas como concesiones por parte del Estado y del Capital. Desde esta visión acotada, según la autora, la política social se caracteriza por:

a. Estar encaminadas a 'corregir' los efectos negativos producidos por la acumulación capitalista; 
b. Con finalidad redistributiva vertida mediante un conjunto sistemático de acciones desde el Estado;

c. Son percibidas como una 'concesión' por parte del Estado; generalmente son definidas desde los poderes estatales;

d. La solución se vislumbra predominantemente desde la adecuada redistribución de la renta para el logro de un denominado 'equilibrio social';

e. $Y$ poseen un carácter paliativo, correctivo y compensatorio.

En tal sentido, y coincidiendo con Young (2011) resulta meritorio expresar que importante es atender no ya a la propia distribución de bienes materiales, sino a los mecanismos por medio de los cuales ésta tiene lugar, así como también, al contexto institucional que le favorece. O sea, que "los conceptos de dominación y opresión, antes que el concepto de distribución, deberían ser el punto de partida para una concepción de la justicia social" (Young, 2011, p. 33). Por ende, el ejercicio de la política social desde una mirada ajustada exclusivamente al lente de la política redistributiva, más que eliminarse o erradicarse la injusticia y opresión, parecería que estas se refuerzan (Palacio, 2011).

En consecuencia, se devela que el paradigma distributivo no contemplaría el análisis de las estructuras y relaciones sociales. Con ello, podría verse capturada en la predilección de las cuestiones de tipo práctico en detrimento de aquellas de tipo críticas, analíticas y reflexivas. Siendo estas últimas fundamentales en aras de la justicia social que, por ser de carácter simbólico y cultural, no pueden reducirse a asuntos meramente distributivos (Young, 2011). 
De igual forma, el paradigma distributivo no inferiría de los propios límites de su dimensión lógica y discursiva: la idea de la distribución perfecta y cantidad exacta. Pues, si como asevera Rawls (1991), la justicia de un esquema social reside en la 'perfecta' distribución de derechos y deberes, por lo que este paradigma se ocuparía de todo valor social que, en las cantidades 'exactas', alcanzará ser poseído por la sociedad, con total independencia de que nos estemos refiriendo a derechos, oportunidades $\mathrm{o}$, en un caso extremo, a la autoestima y el poder de los individuos.

Entonces, desde esta apreciación distributiva [exclusivamente] resultarían aspectos de la vida social entendidos desde una apreciación determinista de la justicia y sin distinción alguna; no se verían como una vida social a partir de relaciones e interacciones sociales. Por el contrario, sería visto como objetos desde una ontología social fragmentada, así como a un modelo estático de lo social.

Lo anterior, conlleva a una fragmentación de la vida social que despolitiza al sujeto, y que en la confabulación de un crimen de lesa humanidad, reproduce y refuerza un amplio espectro de injusticia y opresión. Situación que se ensaña y recrudece en nuestra realidad caribeña, latinoamericana y porque no decirlo, terráquea.

\section{La opresión y justicia social desde un pensum alternativo: prejuicio + poder}

El Ser, en contraste con el Otro.

La opresión e injusticia social se configura y adhiere cotidianamente a través de normas, acciones, instituciones y 
sistema [no resultará en sorpresa identificar el(los) lugar(es) y función(es) de la política social dentro de estos aspectos]. O sea, transita y se manifiesta a través del lenguaje, símbolos, subjetividades, estructuras, pormencionar algunas dimensiones. Con ello: cataloga, categoriza y [re]ubica la existencia del ser humano en un tiempo y espacio dado; es decir que sobreviene una edificación geohistórica de un ente 'diferente' y edificado "desde posiciones centrales del conocimiento, y de mostrar cómo a través de estas construcciones la conciencia eurocentrista se ha ido constituyendo así misma como el Ser, en contraste con ese otro" (von der Walde, 1998).

Es así que el Otro, tal y como le han denominado Spivak (1998) y Dusell (2009), se ancla particularmente en la experiencia histórica de la colonización, en el establecimiento de una monoidentidad y en una relación de subordinación del Otro. En consecuencia, el entendimiento de este aspecto resulta fundamental para la exploración acá vertida, puesto que descubre a un ser que es cubierto por un sufrimiento producido a partir de la dominación o exclusión manifestada tanto en una estructura, como en la diversidad de la experiencia humana.

Lo anterior, podría verse contextualizado a partir de una realidad latinoamericana, donde a la luz del Ser, el Otro habita 'traumáticamente' en la corporalidad del[a] aborigen, negro[a] o mestizo[a]. A su vez, se concibe a esta persona como una abandonada por la esperanza de no poder ser merecedor de los 'beneficios' o de un 'estilo de vida' que resulta de ser parte de la nación o suelo en supremacía. Connota una realidad caracterizada por naciones y pueblos explotados por el capitalismo avasallador; es la presencia de la mujer oprimida por el patriarcado [donde el hombre es también víctima]; donde lo infanto-juvenil y cultura popular es dominada; y en la evidencia de que grupos de diversidad identitaria son discriminados; entre otros. 
Por lo antes visto, el Otro se encuentra en un confín de opresión e injusticia, puesto que "hay dolor de la corporalidad inmolada, [desemboca en] la negación de la vida y de su simultanea posición asimétrica o excluyente en la no-participación discursiva" (Dussel, 2009 p. 302).

Situado en este punto, se torna pertinente proceder a exponer una aproximación argumentativa que permita visibilizar el amplio espectro de la opresión e injusticia social y su importancia dentro del análisis de las políticas sociales. Asimismo, no resultará ajeno a quien lee que intenta presentar un ejercicio analítico que sobrepase la consideración analítica 'habitual', en este caso en particular, de aquello discurrido exclusivamente desde el paradigma distributivo.

\section{Conjunción prejuicio + poder: una mirada de amplio espectro de la opresión e injusticia social.}

Un primer acercamiento de contenido.

Los esfuerzos para dar una aproximación a la opresión e injusticia social, en cuanto significado y referentes, requiere de una lectura de contextos de alta complejidad y de perspectivas que integren, articulen y problematicen. Aunque resulta curioso que, aunque requiere de diversos y dedicados esfuerzos que permitan visibilizarle, sus efectos se muestran frente a mi nariz [incluso en mi propio ser].

Para tales efectos, recurro a una mirada de la opresión e injusticia social a partir de la conjunción "prejuicio más poder" (Lester y Johnson, 1990, pp. 306-307, según citado por Quiñones, 2007) explicada a partir de teorías críticas y postcoloniales. Con ello, se posibilita un estudio a partir de un 
contexto caracterizado por la dominación y exclusión, y que en su interacción configuran ese Ser en contraste con el Otro. Es decir, permite visibilizar diversos espacios de entrecruce donde ocurre la intersección de [inter]subjetividades, lenguajes, símbolos, estructuras y relaciones de poder.

En tal sentido, se presenta esta óptica combinada donde "convergen el racismo, clasismo, sexismo, heterosexismo, eurocentrismo [entre otros]" (Quiñones, 2007, p. 76). O sea, permite hacer visible y vocalizable aquello que se empeña en negar la vida y que rechaza el reconocimiento de la diversidad de la experiencia humana a partir del prejuicio y la multidimensionalidad del poder.

No obstante, la anterior argumentación podría suscitar la interrogante de ¿Por qué recurrir desde el Trabajo Social a una teorización ajustada al lente de lo postcolonial y teorías críticas para pensar la opresión e injusticia social? Sin duda alguna, este cuestionamiento junto con muchas más surgirá a la luz de quien sirve de esta lectura. No obstante, confirmo humildemente que a través de este análisis se podrá reflexionar sobre cómo a partir de la intersección de las dimensiones de la vida humana, situamos a las personas en los discursos ideológicos y en la política social. O sea, una mirada alterna permite, en un sentido amplio, construir una herramienta reflexiva y crítica que gire en torno a los valores ideológicos del Trabajo Social, esto es: la justicia social, emancipación de todo tipo de opresión y libertad para la autodeterminación, por mencionar algunos.

De igual forma, será de beneficio considerar esta óptica como una alternativa que alienta y que "[...] cambia los criterios para redefinir las preguntas o las maneras de ver las cosas. Exhibe la apertura de todo, puesto que no se puede 
dar por sentado los valores estéticos, las dimensiones [inter] personales, referentes binarios [...]" (Maldonado, 2015).

\section{El prejuicio a luz de la interseccionalidad: múltiples ubicaciones e intensidades de opresión}

En relación al prejuicio.

De forma introductoria y groso modo, por prejuicio [en el sentido que acá se presenta] se entenderían variados discursos, ideas y acciones de distinción a partir de la estigmatización según la clase, origen, género, sexo e ideologías, por citar algunos. Simultáneamente incluye dimensiones que se esbozan a partir del pensar [aspecto cognitivo], del sentir [componente emotivo] y la actuación [elemento conativo]. Es decir, cuándo veo a una persona inmigrante o alguien con diagnóstico de esquizofrenia ¿qué pienso? ¿qué siento? y ¿cómo actúo?

Según Young (2011), el prejuicio se ve contextualizado por injusticias que son posibilitadas y reproducidas por las prácticas cotidianas, las que incluso podrían ser vistas a primera mano, como inocentes o sin intención. Esto se ejemplifica mediante la generalización de: las personas pobres con la ignorancia, la delincuencia y el mantengo; a ricos con la soberbia y la prepotencia; las madres solteras con conductas irresponsables; el padre como proveedor del hogar; que las personas blancas son más bellas y no tienen pelo malo; todos los de ese país son terroristas o tontos, entre otros estigmas.

De igual forma, esta misma autora advierte que también el prejuicio incluye procesos institucionales que de manera sistemática, 
[...] impiden a algunas personas aprender y usar habilidades satisfactorias y expansivas en medios socialmente reconocidos, o [mediante] procesos sociales institucionalizados que anulan la capacidad de las personas para interactuar y comunicarse con otras o para expresar sus sentimientos y perspectivas sobre la vida social (Young, 2011, p. 68).

En tal sentido, el movimiento conceptual de prejuicio que se intenta abordar de acá en adelante, direccionará a vincularle con múltiples maneras de interacción e intersección entre las categorías social y culturalmente construidas en un espacio-tiempo dado. Se ilustrará que esa confluencia multidireccional y que ocurre en simultáneos niveles, contribuye a una sistemática injusticia social y opresión, mismos que toman forma en la configuración delas diversas experiencias de la vida humana.

\section{En relación a la interseccionalidad.}

Actualmente, la noción de interseccionalidad ya no debe representar 'un pie de página' dentro del análisis de la opresión e injusticia social. Es decir, su edificación continua por más de cinco décadas (Thompson, 2002), ha permitido situarle dentro de formulaciones teóricas, paradigmáticas y en esquemas interpretativos a partir de la de la práctica en la vida humana.

De igual forma, leer y comprender la interseccionalidad precisa a urdir en elementos iniciales que provocaron su desarrollo y propia expansión reflexiva. En tal sentido, Crenshaw (1989) acompaña la definición de este concepto a través del análisis de diversas maneras en que la raza y género 
esculpían distintitas dimensiones de vida de ciertas mujeres negras. Es decir, surge la conceptualización de que el amplio espectro de las experiencias humanas no se supedita a unos límites 'tradicionales' de discriminación por raza o género, dado que en la intersección del racismo y sexismo, esta práctica de opresión e injusticia social no puede ser capturada totalmente mirándoles por separado.

Es importante mencionar que, aunque la noción de interseccionalidad se inició prácticamente desde la exploración entra la raza y género, esta se ha logrado expandir en asuntos como clase, etnicidad, identidad de género, nacionalidad, discapacidad, ideología, entre otros. O sea, se entiende que ocurren múltiples y posibles encuentros de aspectos en una persona. A su vez, estas distintas configuraciones y formas de la opresión e injusticia social no solo están interrelacionadas, sino que son unidas, influidas y formadas por, unas a las otras (Crenshaw, 1991).

Es decir, la opresión se efectúa en diversas formas y se despliega en distintos grados de intensidad (Hill Collins, 2000). A manera de ejemplo, la opresión ocurre en una configuración e intensidad particular según si es un hombre negro, heterosexual pobre o en un hombre negro, homosexual de clase alta.

De la misma manera, es de suma importancia y beneficio auscultar en la densidad teórica de la opresión e injusticia social a partir de la interseccionalidad. Ya que en esta tarea se podrá visibilizar una serie de dominios que emergen en la sociedad y que se manifiestan a través de las dimensiones interpersonales, de relaciones de poder, de estructuras, de disciplina y hegemonía.

En tanto, se requerirá de esfuerzos adicionales para el estudio de dicha organización total de dominio, puesto que 
es un asunto que interseca lo expuesto anteriormente en relación al prejuicio y la interseccionalidad. Esto ha sido abordado desde perspectivas de diversos autores y ángulos complementarios. A saber, en la matriz de poder y dominación por Hill Collins (2000), en la matriz colonial a partir de Quijano (2000b) y Mignolo (2000), y a través de saberes modernos Foucault (1998). Es precisamente a partir de la configuración del poder y dominación, que se posibilita un acercamiento a diversas características de las categorías sociales que son definidas a partir de un sistema mundo colonial/moderno.

\section{El Poder a la luz del sistema mundo colonial/moderno.}

Tal y como se ha mencionado anteriormente, adicional al asunto del prejuicio se encuentra el poder. En este sentido, se torna pertinente mencionar que precisamente a partir del pensamiento de Gramsci, específicamente lo relacionado a la triada raza/etnia/clase, se visibiliza la importancia de los aspectos hegemónicos, históricos y culturales dentro del estudio los fenómenos sociales. A su vez, estos son asuntos que no han cesado de problematización, trasladándose actualmente al debate dominación/explotación/conflicto según Quijano (2014).

En tal sentido, Gramsci (2011) atisbó una formulación teórica rica y compleja sobre la dominación y la coerción que mantiene la supremacía de unas personas sobre otras. Es decir, su atención giró en torno a que

[...] la diversificación de los antagonismos sociales, la 'dispersión' del poder que se da en las sociedades, la hegemonía se sostiene no solo por medio de la 
instrumentalidad impuesta por el Estado, sino que [también] se basa en las relaciones e instituciones de la sociedad civil" (Hall \& Giraldo, 2005).

En consecuencia, emerge desde un primer plano una nueva dimensión del poder. Uno que sugiere que éste fluye a través de las instituciones 'voluntarias' e instituciones de la sociedad civil y que conforma un sujeto colectivo: es decir en la escolarización, la familia, la economía, las iglesias, el Estado. También, entrevé que discurre mediante las relaciones 'privadas' para la configuración de la subjetividad: o sea, en las identidades de género, sexuales, étnicas, culturales entre otras. Es así que lo anterior sugiere la existencia de asuntos vinculados a la hegemonía y supremacía respecto al poder, el saber y ser.

Es por tal motivo que, para dar aproximación a la dimensión del poder, recurro al pensar post-colonial. Es a partir de esta óptica que se genera un cuestionamiento del efecto de la colonialidad del poder, saber y ser a partir de una configuración del sistema-mundo. En este sentido, por colonialidad se hará referencia a la involuntariedad, dominación, alienación y asimetría de estructuras políticas, injusticia social, exclusión y marginación geopolítica en las configuraciones del poder, saber y ser (Quijano, 2000a).

Estas tres clasificaciones, se pueden explicar mediante: a) como la clasificación social sobre la idea de la raza -denominado como la colonialidad del poder-; b) relaciones del conocer: racionalidad técnico-científica -colonialidad del saber; y c) relaciones del Ser: experiencia vivida y no solamente la corporalidad -colonialidad del ser-. En tal sentido, y de manera similar a lo abordado en a partir de la interseccionalidad, estas relaciones no conforman dimensiones separadas $\mathrm{O}$ 
fragmentadas, sino que por el contrario, se relacionan y fortalecen (Quijano, 2000a).

Ubicado en este apartado particular y para efectos ilustrativos, recurro al abordaje de uno de estos tres factores. En este sentido, Quijano (2000b) establece que, mediante la colonialidad del poder, se abrió paso para que, a partir de la teorización de la idea de la raza, se ocasionara la clasificación social y la re-identificación histórica en una distorsionada ubicación temporal a partir del año de 1492.Ocurre que a partir de esta fecha se inició la conquista y el ejercicio de una reidentificación histórico-espacial de América. Es decir, a partir de esta perspectiva se articuló e incorporó toda forma de opresión dentro del tejido constitutivo de la modernidad.

Este proceso de constitución anterior, resulta en la advertencia de Quijano (2000b), quien establece que no es posible comprender la modernidad sin la colonialidad, y viceversa. Es decir, la modernidad y colonialidad aparecen como las dos caras del patrón mundial de poder actualmente vigente, es un modo colonial/moderno, eurocentrado, lo cual es configurado mundialmente a partir del capitalismo.

Así, la modernidad se presenta como la 'cara ilustrada', la que incluso es considerada como el proceso de la creciente racionalización de la vida social. La 'otra cara', la menos reluciente y que normalmente es ocultada, es la colonialidad.

Respecto a lo anterior, se hace referencia a las relaciones de poder que se establecieron entre lo europeo y lo no europeo sobre la base de la idea de 'raza'. Emergen supuestas diferencias biológicas entre los seres humanos que hacen a unos superiores -los conquistadores [el Ser]- y a otros inferiores -los colonizados [el Otro]. 
Como se puede observar, se expone un referencial teórico que visibiliza la existencia de "un modelo hegemónico global de poder localizado desde de la Conquista [...], y que combina dispositivos para la dominación colonial y de las estructuras de relaciones sociales" (Moraña, Dussel y Jáuregui, 2000, p. 19). Es decir, ocurre un ejercicio de la opresión desplegado por la modernidad a través de los dispositivos de la colonialidad del poder, del ser y saber en un sistema mundo colonial/moderno. A su vez, sus efectos se conjugan, refuerza y recrudecen en la diversidad de la experiencia humana que emerge a partir de las múltiples maneras de interacción e intersección entre las categorías social y culturalmente construidas en un espaciotiempo dado.

\section{Puntualizando en algunas reflexiones}

En primera instancia creo que...

Un enfoque como el que aquí propongo, será inteligible si se expone a la consideración de que la opresión e injusticia social es [re]producida a partir de diversas dimensiones interseccionales de la vida humana y configurada en un entramado de poder global colonial/moderno.

En tal tarea, resulta fundamental considerar como hilo conductor y reflexivo las dimensiones del prejuicio y poder. $\mathrm{O}$ sea, esta conjunción teórica y de la práctica política impulsa un esfuerzo para lograr un cambio de paradigma de cómo se desarrolla el análisis de las políticas sociales desde el lente del Trabajo Social.

Se ha tratado de dilucidar que tanto el análisis de la opresión e injusticia social desde el sistema mundo que configura un 
patrón de poder mundial, como desde la interseccionalidad, se reposicionan elementos adicionales en comparación al análisis 'convencional' de opresión. Es decir, analizar las relaciones de tipo colonial/moderno es fundamental, pero también es importante reconocer la existencia de las múltiples ubicaciones e intensidades de la opresión, las cuales tiene que ver con género, raza, edad, etnia, clase, nacionalidad, sexualidad. Esto nos posibilita preguntarle cara a cara a la modernidad y viabiliza poder retirar ese manto que oculta el aparente y único rostro.

De igual forma, se expresa que la opresión desde la perspectiva postcolonial y crítica no obedece a romanticismos latinoamericanos. Obedece a puntos de enunciación que narran la experiencia desde lo que oprime, por ejemplo, desde Centroamérica, desde el Caribe, desde Latinoamérica. En tal sentido, se confirma una de las tareas del Trabajo Social en un primer plano: desnaturalizar las estructuras opresoras [internas y externas], las cuales recrudecen y sostienen las situaciones con las que día a día enfrentamos: violencia, discrimen, deshistorización, desigualdad, prejuicios, vulneración, políticas sociales acotadas, entre otras.

\section{Ante una posibilidad de resistencia y [trans]formación.}

Es a raíz de la auscultación epistemológica y a la consistente negación e insistencia de relegar a la razón distributiva como la única, exclusiva conocedora y obradora de la justicia social y opresión, que se hace visible lo aparentemente invisible. Es bajo la crisis de aquello relacionado al paradigma distributivo que actúa bajo lo normativo y mecanicista, que se origina el cuestionamiento sobre ¿cuál es el sustento teóricometodológico de las propias reflexiones desde la teoría? 
¿las teorías, qué respuestas ofrecen a los desafíos sociales y políticos? ¿acaso la naturaleza ontológica no reconoce la posibilidad del saber desde los distintos puntos de la experiencia vivida?

De igual forma, surge la necesidad del establecimiento de densos puentes de comprensión con el mundo lo social. Se debe instar a plantar cimientos para desarrollar y consolidar fundamentos desde una perspectiva crítica, postcolonial y en la complejización de las ópticas a través del conocimiento, distinción, selección y organización intelectual. Las teorías o enfoques presentados en este documento, ofrecen una oportunidad para generar un juicio que va desde la comprensión abstracta hasta llegar a factores concretos, lo que permite re-significar las falacias, errores, fricciones y encuentros con la sociedad, esto según lo hace saber Husserl (1992).

Consecuentemente, se evidencia lo importante que resulta establecer espectros que medien y transformen el sentido entre el pensamiento reflexivo, lenguaje crítico y acción creadora. Es vital generar y promover una movilidad consistente y visible sobre la teoría y la praxis: el cómo saber y cómo hacer la praxiología hacia un Trabajo Social portador de atributos disciplinarios, reflexivos, críticos y propositivos que articulen corrientes de pensamiento y perspectivas teóricas alternas, como en lo vinculado a los servicios y políticas sociales. A esto, bajo el lente de Gramsci (1951), se le denominaría el ejercicio de una acción mentada y sensible.

Finalmente, espero que lo anteriormente expuesto pueda convertirse en algo que aspire e inspire a la expansión de más interrogantes y esfuerzos para lograr mayor comprensión sobre ello. Para esto, será clave la consideración de propiciar una ruta dialógica en tal intención. 


\section{Referencias}

Crenshaw, Kimberle. (1989). Demarginalizing the Intersection of

Race and Sex: Black Feminist Critique of Antidiscrimination Doctrine, Feminist Theory and Antiracist Politics. University of Chicago Legal Forum, 139-167.

Crenshaw, Kimberle. (1991). Mapping the Margins: Intersectionality, Identity Politics, and Violence Against Women of Color. Stanford Law Review, 1241-1299. Recuperado de: http://socialdifference.columbia.edu/files/ socialdiff/projects/Article__Mapping_the_Margins_by_ Kimblere_Crenshaw.pdf.

Dussel, Enrique. (2009). Ética de la liberación en la edad de la globalización y de la exclusión. (6ta. ed). España: Trotta.

Foucault, Michel. (1988). El sujeto y el poder. Revista Mexicana de Sociología, Vol. 50, No. 3 (Jul-Sep) 3-20 Recuperado de http://www.jstor.org/stable/3540551.

Fraser, Nancy. (1995). From redistribution to recognition? Dilemas of Justice in a postsocialist. New Left Review, 68-93.

Gramsci, Antonio. (1951). Passato e presente in Opere di Antonio Gramsci. Vol. 7. Turin: Einaudi.

Gramsci, Antonio. (2011). Prissions notebook (Vol. 1, 2 \& 3). Columbia University Press.

Hall, Stuart. \& Girlado, Santiago. (2005). La importancia de Gramsci para el estudio de la raza y etnicidad. Revista Colombiana de Antropología, 219-257 Recuperado de http://www.scielo.org.co/scielo.php?script $=$ sci_ arttext\&pid=S0486-65252005000100008\&lng=en\&tIng=es.

Hill Collins, Patricia. (2000). Black feminist thought: knowledge, consciusness and the politics of empowerment (2da. Edición). Obtenido de Feministes-radicales-org: www. feministes-radicales.org/wpcontent/uploads/2010/11/ Patricia-Hill-Collins Black Feminist Thought Knowledge 
Husserl, Edmund. (1992). Ideas relativas a una fenomenología pura y a una filosofía fenomenológica. México : Fondo de cultura económica.Paidós.

Maldonado, Nelson. (2015). (Notas de seminario en el que participé). (FILO9095_Introducción al giro decolonial). San Juan: Notas en posesión del que suscribe.

Mignolo, Walter. (2000). La colonialidad a lo largo y a lo ancho: el hemisferio occidental en el horizonte colonial de la modernidad. En E. Lander, La colonialidad del saber: eurocentrísmo y ciencias sociales. Perspectivas Latinoamericanas (págs. 56-86). Buenos Aires: Consejo Latinoamericano de ciencias sociales (CLACSO).

Moraña, Mabel, Dussel, Enrique, \& Jáuregui, Carlos. (2000). Colonialism and its Replicants. En Coloniality at Large: Latin America and the Postcolonial Debate (págs. 1-20). Londres: Duke University Press.

Palacio Ricondo, Tamara. (2011). Justicia y diferencia en Iris Marion Young. Eikasia. Revista de filosofía, 74-106.

Pastorini, Alejandra. (1999). ¿Quién mueve los hilos de las políticas sociales? Avances y límites en la categoria "concesión-conquista". En E. Borgianni, \& C. Montaño, La política social hoy (págs. 207-232). Sao Pablo: Cortez Editora.

Quijano, Aníbal. (2000a). Colonialidad del poder: Cultura y conocimiento en América Latina. En W. Mignolo, Capitalismo y geopolítica del conocimiento: El eurocentrismo y la filosofía de la liberación en el debate intelectual contemporáneo (págs. 117-131). Buenos Aires: Ediciones del Signo.

Quijano, Aníbal. (2000b). Colonialidad del poder, eurocentrismo y América Latina. En E. Lander, La colonialidad del saber: eurocentrismo y ciencias sociales. Perspectivas Latinoamericanas (págs. 201-242). Buenos Aires: Consejo Latinoamericano de Ciencias Sociales (CLACSO). 
Quijano, Aníbal. (2014). Colonialidad del poder y clasificación social. En B. Sousa Santos, \& M. P. Meneses, Epistemologías del Sur (Perspectivas) (págs. 67-143). Madrid: Ediciones Akal.

Quiñones Rosado, Raúl. (2007). Consciousness-inAction: Toward and Integral Psychology of Liberation \& Transformation. Caguas: ilé publication.

Rawls, John. (1991). Justicia como equidad. México: Fondo de cultura económica.

Spivak, Gayatri C. (1998). Postestructuralism, Marginality, Poscoloniality and Value. En P. Cllier, \& H. (. Geyer Ryan, Literary Theory Today (págs. 219-245). New York: Polity Press.

Thompson, Becky. (2002). Multiracional Feminism: Recasting the Chronology of Second Wave Feminism . Feminist Studies, 336-360.

von der Walde, Erna. (1998). Realismo mágico y poscolonialismo: construcciones del otro desde la otredad. En S. Castro-Gómez, \& E. e. Medieta, Teorías sin disciplina (latinoamericanismo, poscolonialismo y globalización en debate). México: Miguel Ángel Porrúa.

Young, Iris M. (2011). Justice and the politics of difference. Princeton, N.J.: Princenton University Press. 